\title{
Biomolecular patterning of glass surfaces via strain- promoted cycloaddition of azides and cyclooctynes $\dagger$
}

Received 25th October 2013 Accepted 13th December 2013

\author{
M. A. Wijdeven, ${ }^{a}$ C. Nicosia, ${ }^{b}$ A. Borrmann, ${ }^{a}$ J. Huskens ${ }^{b}$ and F. L. van Delft ${ }^{\star a}$
}

DOI: $10.1039 / c 3 r a 46121 a$

www.rsc.org/advances

Metal-free, strain-promoted alkyne-azide cycloaddition (SPAAC) is employed as a versatile technology for the modification of glass with biomolecules. Patterning is executed by stamping of a fluorogenic azidocoumarin or a cyclooctyne to the glass surface, to obtain a unique anchor point for subsequent functionalization by SPAAC. The azidocoumarin at the same time enables straightforward fluorescent read-out of surface reactions. A strong increase in fluorescence is indeed observed upon metal-free reaction with two readily available cyclooctynes, BCN or DIBAC. In addition, functionalized BCN derivatives are employed for glass surface patterning with biotin or even a 27 kDa protein (green fluorescent protein), upon simple incubation.

Surfaces containing immobilized proteins or peptides, for example used in protein or peptide biochips, offer a wide range of applications varying from diagnostic tests to biomarker discovery. ${ }^{1}$ However, the preparation of biochips can be challenging due to the potentially sensitive nature of peptides and proteins. Most commonly applied immobilization strategies rely on aspecific techniques involving either physisorption, or random covalent attachment, via side-chains to a functionalized surface. ${ }^{2}$ In this way effective immobilization is obtained, although, due to the lack of orientational control during conjugation, diminished binding activity can lead to false negative results. As a consequence, increasing interest has been focused on site-specific ligation based on the introduction of a non-native functionality into peptide and protein structures as an anchor point for conjugation. ${ }^{3,4}$ A seminal contribution in this field of bioorthogonal ligation is the Staudinger ligation, performed with an immobilized phosphane group and an azidelabeled peptide or protein. ${ }^{5-7}$ Another example entails the

\footnotetext{
${ }^{a}$ Institute for Molecules and Materials, Radboud University Nijmegen, Heyendaalseweg 135, 6525 AJ Nijmegen, The Netherlands. E-mail: f.vandelft@science.ru.nl

${ }^{b}$ Molecular Nanofabrication group, MESA+ Institute for Nanotechnology, University of Twente, P.O. box 217, 7500 AE Enschede, The Netherlands

$\dagger$ Electronic supplementary information (ESI) available: Experimental details including synthetic procedures, surface modification procedures and additional analysis. See DOI: 10.1039/c3ra46121a
}

photoactivatable immobilization of biomolecules to surfaces based on the selective reaction of a free thiol with an alkene or alkyne, the so-called thiol-ene reaction. ${ }^{\mathbf{8} 9}$ Arguably the most applied technology for peptide or protein conjugation, outside traditional amide bond formation, is based on the coppercatalyzed azide-alkyne cycloaddition (CuAAC). However, the indispensible use of toxic $\mathrm{Cu}(\mathrm{I})$ may be disadvantageous for certain biological applications. ${ }^{\mathbf{1 0 , 1 1}}$

The requirements for controlled ligation, i.e. fast, specific and selective reaction under mild conditions, is perfectly fulfilled by strain-promoted cycloadditions. Such cycloadditions, based on the high reactivity of ring-strained alkenes and alkynes, are now commonly recognized as versatile tools for selective bioconjugation in solution, in particular within a chemical biology context. ${ }^{12,13}$ In addition, some applications of strain-promoted alkyne-azide cycloaddition (SPAAC) for surface modification have been reported. ${ }^{14-19}$ Because SPAAC can serve such a wide range of applications, the search for the optimal balance between reactivity, stability and water solubility of the cyclic alkyne is continuously driving the research on strainpromoted cycloadditions. After Bertozzi and co-workers first recognized the potential of cyclooctynes for bioconjugation, several other analogues with improved features have been developed, ${ }^{20,21}$ with contributions from our own group involving BCN (bicyclo[6.1.0]nonyne, 1) and DIBAC (dibenzoazacyclooctyne, 2, Fig. 1A). ${ }^{22,23}$ While DIBAC combines high stability with exceptional reactivity with aliphatic azides in particular, $\mathrm{BCN}$ on the other hand is exceptionally reactive with tetrazines. ${ }^{24}$ In addition, BCN has the advantage of being less lipophilic, hence more suitable for application in aqueous environments, and significantly easier to synthesize.

Here we report a strategy for the local functionalization of glass surfaces with biomolecules based on SPAAC with BCN and DIBAC (Fig. 1B). In order to obtain patterns, reactive microcontact printing $(\mu \mathrm{CP})^{25}$ of either an azide or a cyclooctyne is explored. Subsequently, several relevant biomolecules were readily introduced via SPAAC without the need of additives, and corroborated by fluorescence measurements. 
A<smiles>[R]CC1[C@H]2CCC#CCC[C@H]12</smiles>

$\mathrm{BCN}$ 1 a $\mathrm{R}=\mathrm{H}$ b $\mathrm{R}=\mathrm{COOSu}$

B



Fig. 1 (A) Building blocks for SPAAC. (B) Concept of two-stage glass modification via strain-promoted cycloaddition of azide and cyclooctyne (SPAAC).

We set out to explore the modification of glass plates by a two-stage strategy involving first the covalent attachment of an azide or a cyclooctyne, followed by SPAAC to introduce the biomolecular functionality of interest, covalently attached to a cyclooctyne or an azide (Fig. 1B). In order to monitor the progress of the SPAAC surface modification in a straightforward manner, the use of a fluorogenic substrate, such as coumarin 3 (ref. 26) (Fig. 1A), was considered preferential, because falsepositive results due to non-covalent interactions should be absent. ${ }^{27,28}$ As expected, subjection of a solution of coumarin ester 3a to SPAAC with bicyclononyne (BCN, 1a) led to strong $406 \mathrm{~nm}$ fluorescence upon excitation at $340 \mathrm{~nm}$ (see ESI $\dagger$ for details).

Subsequently, glass slides modified with a TPEDA monolayer, prepared according to a literature procedure, ${ }^{29}$ were patterned with the azidocoumarin $\mathbf{3 b}$ using reactive microcontact printing $(\mu \mathrm{CP})$ (Scheme 1). To this end, non-oxidized PDMS stamps were inked with the active ester derivative of coumarin (3b) and applied onto the TPEDA-glass slide, affording functionalized glass type $\mathbf{A}$ by standard amide bond formation.

Alternatively, the TPEDA-glass was treated with the $\mathrm{BCN}$ derived active carbonate $\mathbf{1 b}$ (not depicted), however due to the fact that the latter BCN-containing surface turned out to be of low stability and reproducibility (see ESI, Fig. S6†), we have focused entirely on the versatility of the azide-containing surface type $\mathbf{A}$ in subsequent studies.

Having the azido-functionalized glass plates in hand, we were ready to explore the on-glass SPAAC modification. Thus, the azido-coumarin-containing glass surface type A was initially incubated in a solution of non-functionalized BCN (1a), yielding the expected formation of triazole-linked surface A-1a, as established by the strong increase of fluorescence already after 20 min with the expected pattern (Fig. 2A). The clear pattern showed the functionalized, fluorescent regions containing the triazole-linked coumarin-BCN conjugate, while the black lines have only the amine functionality. Prolonged incubation times gave no further increase in fluorescence, indicating a high degree of functionalization in an efficient manner already after
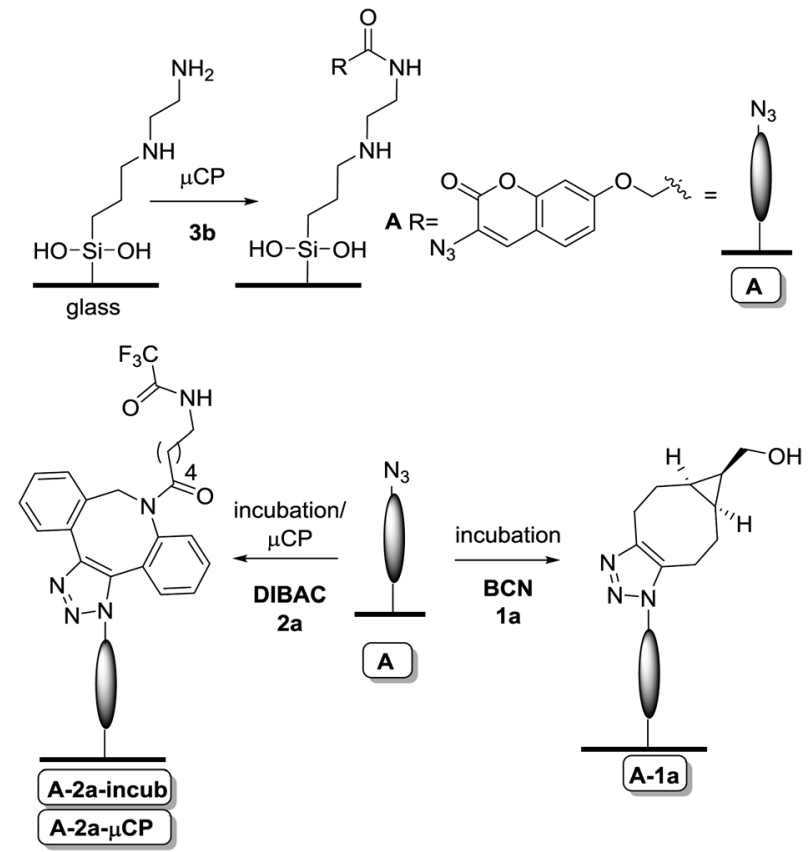

Scheme 1 Reactive $\mu C P$ of coumarin 3b onto TPEDA-functionalized glass, followed by incubation with a cyclooctyne (BCN 1a or DIBAC 2a).
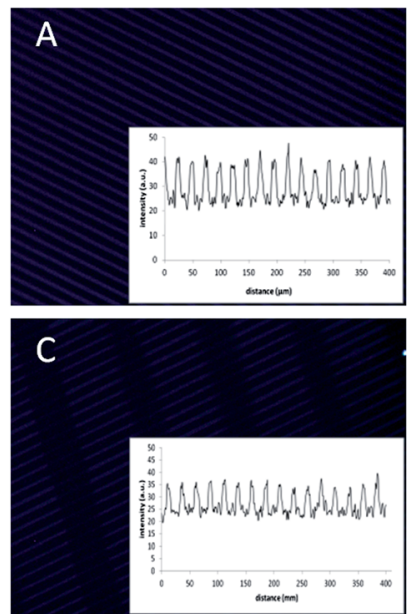

D

Fig. 2 (A) surface A-1a: coumarin slides incubated with BCN (1a) (mag. $=10 \times, 5 \times 15 \mu \mathrm{m}$ lines) (B) surface A-2a-incub: coumarin slides incubated with DIBAC (2a) (mag. $=10 \times, 5 \times 15 \mu \mathrm{m}$ lines) (C) surface A2a- $\mu$ CP: coumarin slides $\mu$ CP with DIBAC (2a) (mag. $=10 \times, 5 \times 15 \mu \mathrm{m}$ lines, $100 \times 100 \mu \mathrm{m}$ lines) (D) platform A, blank (magnification $=20 \times$, $20 \times 5 \mu \mathrm{m}$ lines). Fluorescence microscope pictures after $20 \mathrm{~min}$ incubation (inset is the intensity profile, exposure time $=500 \mathrm{~ms}, \lambda_{\mathrm{ex}}=$ $\left.340 \mathrm{~nm}, \lambda_{\mathrm{em}}=420 \mathrm{~nm}\right)$.

20 min. In addition, XPS analysis at the different stages of functionalization gave another proof because the $\mathrm{C} / \mathrm{N}$ ratio changed clearly and even more indicative was the presence of fluor in the XPS analysis of the last step due to the use of a fluorcontaining BCN derivative in this case (see ESI, Fig. S5†).

Similarly, incubation of surface A with a solution of DIBAC (2a) gave the triazole-modified surface A-2a-incub. In this case 
the fluorescence intensity increase upon functionalization was less (about half) in comparison to incubation with BCN, which is attributed to the increased steric bulk of DIBAC in combination with its lower reactivity for aromatic azides, versus aliphatic azides, ${ }^{30}$ thereby preventing dense packing. The lack of residual accessible azides after incubation with DIBAC was corroborated by the fact that subsequent incubation with BCN did not lead to further increase in fluorescence intensity (see ESI, Fig. S7†). In addition, instead of incubation, orthogonal reactive $\mu \mathrm{CP}$ of DIBAC (2a) with a different pattern on the printed coumarin surface, showed a similar intensity with the desired pattern (surface $\mathbf{A}-\mathbf{2} \mathbf{a}-\boldsymbol{\mu} \mathbf{C P}$ ).

Having established the conceptual validity of SPAAC on glass, we set out to explore bioconjugation events on a surface. In particular, we were interested to explore the functionalization with biotin, for subsequent detection by biological interaction with streptavidin, and the covalent attachment of a GFP, an inherently fluorescent protein, in a specific manner. To this end, patterned azidocoumarin surface type $\mathbf{A}$ (ref. 31) was first incubated with BCN-biotin 4 in methanol for 30 min, leading to glass surface A-4 (Fig. 3D).

As above, generation of blue emission upon incubation with BCN-biotin 4 confirmed the on-glass triazole formation and thus the presence of triazole-linked biotin on the surface (Fig. 3A). At this stage, in the absence of labeled streptavidin, no green fluorescence was observed. Next, the presence of biotin on the surface was investigated by exposure to streptavidin labeled with AlexaFluor488 (AF488), followed by fluorescence microscopy analysis. Indeed, the clear localization of green emission (Fig. 3B) after incubation with streptavidin-AF488 can be taken as an unequivocal indication of the presence of biotin and its availability for the biomolecular interaction with streptavidin. The specificity of the biotin-streptavidin interaction was further proven in a control experiment by subjecting glass-
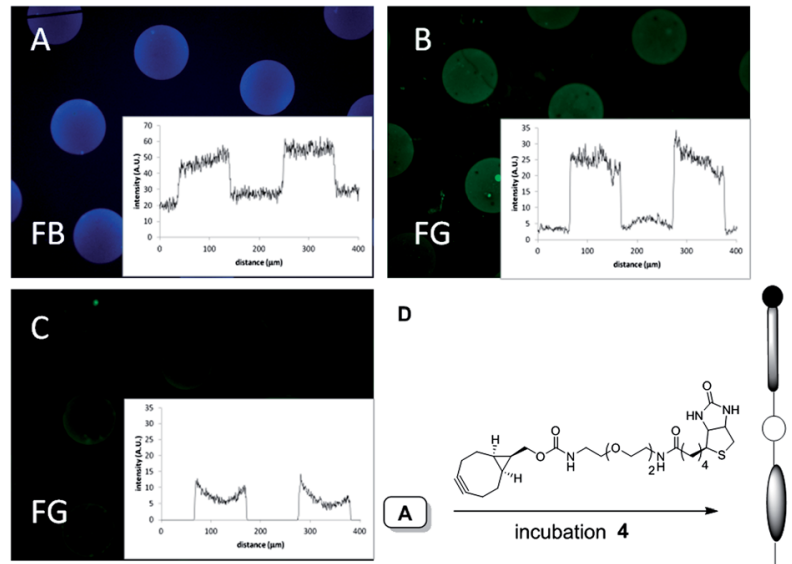

(A-4)

Fig. 3 (A and B) Fluorescence microscope images after incubation of platform A-4 with streptavidin AF488 (inset: intensity profile, gain 2.3). (C) After incubation with streptavidin AF488 and biotin (gain 3.9) Magnification $20 \times, 100 \mu \mathrm{m}$ dots, exposure time $=500 \mathrm{~ms}$. Filter sets: FB: $\lambda_{\mathrm{ex}}=340-380 \mathrm{~nm}, \lambda_{\mathrm{em}}=425 \mathrm{~nm}, \mathrm{FG}: \lambda_{\mathrm{ex}}=450-490 \mathrm{~nm}, \lambda_{\mathrm{em}}=$ $515 \mathrm{~nm}$. (D) Modification of coumarin surface A with BCN-biotin 4 followed by a streptavidin assay.


Fig. 4 ( $A$ and B) Fluorescence microscope images after incubation of platform A with 5 (inset: intensity profile). Magnification 20×, $100 \mu \mathrm{m}$ dots, exposure time $=500$ ms. Filter sets: FB: $\lambda_{\mathrm{ex}}=340-380 \mathrm{~nm}, \lambda_{\mathrm{em}}$ $=425 \mathrm{~nm}$, FG: $\lambda_{\mathrm{ex}}=450-490 \mathrm{~nm}, \lambda_{\mathrm{em}}=515 \mathrm{~nm}$. (C) Modification of coumarin surface A with GFP-BCN 5 .

plate type A (bearing solely azidocoumarin) to the same incubation conditions with streptavidin-AF488. Indeed, both blue and green fluorescence were negligible in this case (see ESI, Fig. S8 $\dagger$ ). Incubation of the biotin-containing glass plates (A-4) to a solution containing biotin and streptavidin (Fig. 3C) also showed fluorescent patterns although with a strongly reduced intensity (about 33\% of B).

Recently, the introduction of BCN in GFP via genetic encoding of unnatural amino acid 6 was described by us, ${ }^{32}$ as well as others. ${ }^{33}$ The site-specific introduction of BCN into GFP by a generic protocol like genetic encoding is serving here as a show-case for the selective and reagent-free protein conjugation to functional azides (Fig. 4C).

Therefore, the azido-coumarin glass plate $\mathbf{A}$ was incubated with GFP-BCN (5) for one hour to afford the GFP-functionalized surface, as confirmed by the blue and green fluorescence microscope pictures (Fig. 4). The fact that the patterning of the surface is not as homogeneous as expected may be explained by partial denaturation of GFP during sonification after the SPAAC reaction. Nevertheless, the intensity and presence of both green and blue fluorescence unambiguously proves the covalent attachment of the protein to the surface.

\section{Conclusions}

We have successfully coated and patterned glass plates with azide or cyclooctyne functional groups, and shown the versatility of such glass plates for further functionalization by SPAAC. In particular, modification of the glass surface with a fluorogenic azidocoumarin and subsequent reaction with either cyclooctyne BCN or DIBAC afforded a straightforward read-out owing to the concomitant formation of a fluorescent linking moiety. The azidocoumarin-containing glass was subsequently 
functionalized with BCN-biotin or with GFP-BCN to show the bioorthogonality of the SPAAC reaction for attachment of sensitive biomolecules to glass. Such functionalized surfaces were used for the detection of biomolecular interactions in water. The latter aspect may find application in a wide variety of areas, such as microarrays and biosensors.

\section{Notes and references}

1 C. Katz, L. L. Beladev, S. Rotem-Bamberger, T. Rito, S. G. D. Rüdiger and A. Friedler, Chem. Soc. Rev., 2011, 40, 2131.

2 P. Jonkheijm, D. Weinrich, H. Schröder, C. M. Niemeyer and H. Waldmann, Angew. Chem., Int. Ed., 2008, 47, 9618.

3 P.-C. Lin, D. Weinrich and H. Waldmann, Macromol. Chem. Phys., 2010, 211, 136.

4 M. Köhn, J. Pept. Sci., 2009, 15, 393.

5 M. B. Soellner, K. A. Dickson, B. L. Nilsson and R. T. Raines, J. Am. Chem. Soc., 2003, 125, 11790.

6 M. Köhn, R. Wacker, C. Peters, H. Schröder, L. Soulere, R. Breinbauer, C. M. Niemeyer and H. Waldmann, Angew. Chem., Int. Ed., 2003, 42, 5830.

7 S. S. van Berkel, M. B. van Eldijk and J. C. M. van Hest, Angew. Chem., Int. Ed., 2011, 50, 8806.

8 C. E. Hoyle and C. N. Bowman, Angew. Chem., Int. Ed., 2010, 49, 1540.

9 P. Jonkheijm, D. Weinrich, M. Köhn, H. Engelkamp, P. C. M. Christianen, J. Kuhlmann, J. C. Maan, D. Nüsse, H. Schroeder, R. Wacker, R. Breinbauer, C. M. Niemeyer and H. Waldmann, Angew. Chem., Int. Ed., 2008, 47, 4421.

10 G. A. Hudalla and W. C. Murphy, Langmuir, 2009, 25, 5737.

11 L. Nebhani and C. Barner-Kowollik, Adv. Mater., 2009, 21, 3442.

12 E. M. Sletten and C. R. Bertozzi, Acc. Chem. Res., 2011, 44, 666.

13 M. F. Debets, S. S. van Berkel, J. Dommerholt, A. J. Dirks, F. P. J. T. Rutjes and F. L. van Delft, Acc. Chem. Res., 2011, 44, 805 .

14 R. K. Manova, S. P. Pujari, C. A. G. M. Weijers, H. Zuilhof and T. A. van Beek, Langmuir, 2012, 28, 8651.

15 R. Manova, T. A. van Beek and H. Zuilhof, Angew. Chem., Int. Ed., 2011, 50, 5428.
16 S. V. Orski, A. A. Poloukhtine, S. Arumugam, L. Mao, V. V. Popik and J. Locklin, J. Am. Chem. Soc., 2010, 132, 11024.

17 A. Kuzmin, A. Poloukhtine, M. A. Wolfert and V. V. Popik, Bioconjugate Chem., 2010, 21, 2076.

18 C. Wendeln, I. Singh, S. Rinnen, C. Schulz, H. F. Arlinghaus, G. A. Burley and B. J. Ravoo, Chem. Sci., 2012, 2479.

19 D. Prim, F. Rebeaud, V. Cosandey, R. Marti, P. Passeraub and M. E. Pfeifer, Molecules, 2013, 18, 9833.

20 N. J. Agard, J. A. Prescher and C. R. Bertozzi, J. Am. Chem. Soc., 2004, 126, 15046.

21 J. C. Jewett and C. R. Bertozzi, Chem. Soc. Rev., 2010, 39, 1272.

22 M. F. Debets, S. S. van Berkel, S. Schoffelen, F. P. J. T. Rutjes, J. C. M. van Hest and F. L. van Delft, Chem. Commun., 2010, 46, 97.

23 J. Dommerholt, S. Schmidt, R. Temming, L. J. A. Hendriks, F. P. J. T. Rutjes, J. C. M. van Hest, D. J. Lefeber, P. Friedl and F. L. van Delft, Angew. Chem., Int. Ed., 2010, 49, 9422.

24 W. Chen, D. Wang, C. Dai, D. Hamelberg and B. Wang, Chem. Commun., 2012, 48, 1736.

25 C. Wendeln and B. J. Ravoo, Langmuir, 2012, 28, 5527.

26 K. Sivakumar, F. Xie, B. M. Cash, S. Long, H. N. Barnhill and Q. Wang, Org. Lett., 2004, 6, 4603.

27 J. Cabanas-Danés, C. Nicosia, E. Landman, M. Karperien, J. Huskens and P. A. Jonkheijm, J. Mater. Chem. B, 2013, 1, 1903.

28 D. Wasserberg, C. Nicosia, E. E. Tromp, V. Subramaniam, J. Huskens and P. Jonkheijm, J. Am. Chem. Soc., 2013, 135, 3104.

29 S.-H. Hsu, D. N. Reinhoudt, J. Huskens and A. H. Velders, J. Mater. Chem., 2008, 18, 4959.

30 Manuscript in preparation.

31 The remaining amines after stamping with coumarin 1 were capped via incubation with acetic anhydride in dichloromethane.

32 A. Borrmann, J. Milles, T. Plass, J. Dommerholt, J. M. M. Verkade, M. Wiessler, C. Schultz, J. C. M. van Hest, F. L. van Delft and E. A. Lemke, ChemBioChem, 2012, 2094.

33 K. Lang, L. Davis, S. Wallace, M. Mahesh, D. J. Cox, M. L. Blackmann, J. M. Fox and J. W. Chin, J. Am. Chem. Soc., 2012, 134, 10317. 